\section{Dinámica del cultivo y mercado de flores en México, 1995-2012}

\section{Dynamics of cultivation and flower market in Mexico, 1995- 2012}

Oscar Eduardo Patrón Osuna*, Fernando Restrepo Betancur, Holmes Rodríguez Espinosa

\footnotetext{
* Magister en Desarrollo Económico Local Universidad Autónoma de Nayarit, Grupo GISER.

** Profesor Titular, Facultad de Ciencias Agrarias. Grupo GISER.

*** $\mathrm{PhD}$ en Agricultura, profesor asistente Grupo GISER. Facultad de Ciencias Agrarias.
}

\section{Resumen}

El objetivo de esta investigación fue analizar la dinámica del cultivo y el mercado de las flores en México en el período 1995-2012. Se efectuó un análisis descriptivo comparativo multidimensional a partir de fuentes de información secundarias. Análisis de componentes principales, análisis descriptivo exploratorio unidimensional y análisis de clúster. La superficie sembrada presentó un incremento significativo $(P<0.05)$ en el último sexenio en alelí, gladiola (gruesa), agapando, ave del paraíso, nardo (gruesa) caso contrario al nardo, gladiola y rosa. El volumen de la producción presentó diferencia estadística a favor del cultivo de nardo entre sexenios. El valor de la producción presentó un aumento a lo largo del tiempo para el cultivo de gladiola (gruesa) caso contrario para el crisantemo y rosa (gruesa) $(P<0.05)$. La producción florícola tuvo un incremento en su participación en la economía del país, convirtiéndose en un subsector con gran potencial de desarrollo.

Palabras clave: Análisis sectorial, floricultura, política agraria, producción de flores.

\begin{abstract}
The purpose of this research was to analyze the dynamics of cultivation and flower market in Mexico in the term 1995-2012. A multi-dimension, descriptive comparative analysis was performed, parting from secondary information sources. Analysis of the main components, singledimension descriptive exploratory analysis, and cluster analysis. The cultivated area showed a meaningful increase $(P<0.05)$ during the last six-year term in alheli, gladiolus, (thick), agapanthus, paradise bird, tuberose (thick), spikenard, gladiolus, and rose.

Volume production showed statistical difference in favor of nard cultivation among such six-year terms. Production figures increased along such period of time for gladiolus (thick) cultivation, opposite to chrysanthemum and rose (thick) $(P<0.05)$. Flower production increase its share in economy of the country, turning into a subsector of a great potential development.
\end{abstract}

Key words: Sector analysis, floriculture, agricultural policy, flower production
Recibido: 11/11/2015

Revisado: 23/02/2016

Aceptado: 01/12/2016

Correspondencia de autor:

oepo39@gmail.com

frbstatistical@yahoo.es

holmes.rodriguez@udea.edu.co

(C) 2016 Universidad La Gran Colombia. Este es un artículo de acceso abierto, distribuido bajo los términos de la licencia Creative Commons Attribution License, que permite el uso ilimitado, distribución y reproducción en cualquier medio, siempre que el autor original y la fuente se acrediten.

Cómo citar:

Patrón, O. E., Restrepo, F., Rodríguez, H. Dinámica del cultivo y mercado de flores en México 1995-2012 UGCiencia 22, 111-118.

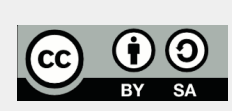




\section{Introducción}

La floricultura a través del tiempo ha demostrado el gran desarrollo que logra traer consigo. En México, según datos de la Secretaria de Agricultura, Ganadería, Desarrollo Rural, Pesca y Alimentación (Sagarpa), se reporta que en la nación en el año 2013 fueron cultivadas 23,088 hectáreas de ornamentales, con valor de producción de 481 millones dólares; en esta actividad participan 25500 productores de flores de corte, plantas en maceta, follaje de corte y follaje de maceta; el mercado ornamental genera 188000 empleos permanentes, 50000 eventuales y más de un millón indirectos. (Sagarpa, 2015). Debido a los cuidados específicos y tiempos precisos que requieren las flores para mantener una calidad óptima, la floricultura se vuelve la única actividad del sector agropecuario capaz de generar empleos permanentes durante todo el año (Santana, 2007).

México es un país con una gran extensión territorial y diversidad agrícola en el cual la floricultura se ha ido incrementando en los últimos años dado que la economía del país se ha diversificado. En el país las condiciones agroclimáticas permiten cultivar alrededor de 349 especies distintas, en un área total estimada de 375000 hectáreas, cerca de 5,8\% se dedica al cultivo de la flor, tanto de ornato como para alimento y uso cosmético; aproximadamente 21,970 hectáreas se destinan a la producción de cultivo ornamentales, de las cuales $52 \%$ (11 424 hectáreas), son cultivadas para producción de flores y follajes de corte (Morales, 2011).

Para el contexto mexicano, no se han reportado en la literatura estudios relacionados con la dinámica del sector florícola; sin embargo, para otros subsectores, el estudio de la dinámica sectorial ha tomado en consideración aspectos como la superficie sembrada, la superficie cosechada, el volumen de la producción y el valor de la producción con el fin de medir la productividad y el comportamiento de la oferta y la demanda (Grammont, 2010).

El análisis de la superficie sembrada y superficie cosechada permite darnos cuenta del riesgo que puede conllevar la floricultura a nivel industrial y los riesgos de pérdidas en la producción de la misma, en cuanto al volumen de la producción debemos asimilar que estos cultivos son vendidos en tallos y gruesas (cada gruesa equivale a 12 docenas o 144 unidades), por lo que es importante conocer la dinámica de variabilidad asociada al valor de producción.

Otros estudios, por su parte, han abordado el análisis de la contribución de las políticas públicas puestas en funcionamiento, por medio de la comparación entre períodos de tiempo, para conocer y comparar su relación con la dinámica de crecimiento y productividad. Adicionalmente, para un mejor entendimiento de las cifras, se decidió analizar el producto interno bruto agropecuario de México y la población nacional para dar cuenta de cómo se encuentra el mercado florícola del país, sus beneficios como actividad económica y la productividad en sí.

Por lo anterior, el objetivo del presente estudio fue evaluar la dinámica de la floricultura en México y la influencia de las políticas agropecuarias por medio de la comparación de los últimos tres sexenios, en relación con el cultivo, producción y comercialización.

\section{Materiales y métodos}

Se efectuó un análisis descriptivo comparativo multidimensional. Se diseñó una base de datos teniendo en cuenta la información reportada por los principales sistemas de información agropecuaria, agrícola y florícola con los que 
cuenta México: Instituto Nacional de Estadística y Geografía de México (Inegi), proyecciones de población del Consejo Nacional de Población (Conapo), información de la Secretaria de Información Agroalimentaria y Pesquera (Siap) y Sistema de Información Agroalimentaria de Consulta (Siacon), teniendo como variables:

\begin{tabular}{|l|l|l|}
\hline Variable & Tipo Variable & Medida \\
\hline Superficie sembrada & Cuantitativa continua & Hectárea \\
\hline Superficie cosechada & Cuantitativa continua & Hectárea \\
\hline Volumen de la producción & Cuantitativa continua & Toneladas \\
\hline Valor de la producción & Cuantitativa continua & Dólares \\
\hline Población nacional & Cuantitativa discreta & Número personas \\
\hline PIB agropecuario nacional & Cuantitativa continua & Dólares \\
\hline Producción nacional de ornamentales & Cuantitativa continua & Dólares \\
\hline Periodo presidencial & Cualitativa & Sexenio \\
\hline
\end{tabular}

Las variables cuantitativas continuas están asociadas a la distribución probabilística normal y las cualitativas al modelo multinomial.

Para el análisis estadístico de los datos, se aplicó el modelo lineal general (GLM), adicionalmente análisis de componentes principales método $\mathrm{R}$, anotando que las variables activas eran de naturaleza cuantitativa asociadas a distribuciones probabilísticas normal. El análisis se complementó por medio de estadística descriptiva unidimensional y análisis de correlación bidimensional por el método de Spearman. Fue utilizando el paquete estadístico SAS University Edition.

\section{Resultados}

En lo referente a la superficie sembrada, se encontró una evolución creciente en el cultivo de Alelí y Gladiola (gruesa); se encontró diferencia estadística $(P<0.05)$ entre sexenios (tabla 1). Para los cultivos de Gladiola, Nardo y Rosa se observó una disminución en la superficie sembrada a lo largo del tiempo $(P<0.05)$. En cuanto a la superficie cosechada para el caso de alelí y gladiola (gruesa) se encontró un incremento detectando diferencia estadística significativa $(P<0.05)$, observando mayor superficie cosechada en el último sexenio. Para el cultivo de gladiola se observó una disminución en la superficie cosechada a lo largo del tiempo $(P<0.05)$. Las otras variedades no tuvieron un cambio significativo $(\mathrm{pH} 0.05)$ en los periodos citados.

Se encontró un incremento creciente de la superficie cosechada en cada sexenio, lo cual indica un aumento en la importancia de la floricultura en los últimos años; paradójicamente, no se ha tenido en el período analizado, una política nacional de fomento al cultivo o exportación de ornamentales, situación que conlleva a pensar que el crecimiento se ha dado por un incremento en la demanda interna de este tipo de productos en el país.

En lo referente al volumen de la producción únicamente el cultivo de Nardo fue el que tuvo aumento con diferencia estadística entre el primer período presidencial con respecto al último sexenio evaluado, a favor de este $(P<0.05)$, en cuanto a Alelí se detectó un pequeño aumento en su volumen de producción, para todos los demás grupos de cultivos no hubo diferencia estadística significativa ( $\mathrm{pH} 0.05)$, en relación con el volumen de producción (tabla 2).

En lo que respecta al valor de la producción se tuvo para el cultivo de Gladiola (gruesa) un aumento en el valor de la misma respecto a lo largo del tiempo presentando diferencia estadística $(P<0.05)$. En cuanto al crisantemo y rosa (gruesa) se observó una disminución en valor de la producción a través de los diferentes sexenios $(p<0.05)$; los demás cultivos se mantuvieron estables $(P>0.05)$. 
Tabla1. Análisis comparativo por sexenio para la superficie cosechada y sembrada asociada a los grupos de cultivos ornamentales.

\begin{tabular}{|c|c|c|c|c|c|c|}
\hline \multirow[b]{2}{*}{$\begin{array}{l}\text { Sexenio } \\
\text { Grupo }\end{array}$} & \multicolumn{3}{|c|}{ Superficie sembrada } & \multicolumn{3}{|c|}{ Superficie cosechada } \\
\hline & $1995-2000$ & $\begin{array}{l}2001-2006 \\
\text { Valor promedio }\end{array}$ & 2007-2012 & $1995-2000$ & $\begin{array}{l}2001-2006 \\
\text { Valor prome dio }\end{array}$ & 2007-2012 \\
\hline $\begin{array}{l}\text { Agapando } \\
\text { (gruesa) }\end{array}$ & $28,0 \mathrm{~b}$ & $52,0 \mathrm{ba}$ & $62,0 \mathrm{a}$ & $28,0 \mathrm{a}$ & $52,0 \mathrm{a}$ & $58,3 \mathrm{a}$ \\
\hline Alelí & $227,0 \mathrm{c}$ & $300,1 \mathrm{~b}$ & $324,5 \mathrm{a}$ & $227,0 \mathrm{c}$ & $300,1 \mathrm{~b}$ & $322,1 \mathrm{a}$ \\
\hline $\begin{array}{l}\text { Ave del paraíso } \\
\text { (gruesa) }\end{array}$ & $33,3 \mathrm{~b}$ & $154,8 \mathrm{ba}$ & $204,5 \mathrm{a}$ & $33,1 \mathrm{~b}$ & 154,6 ba & $198,6 \mathrm{a}$ \\
\hline Clavel (gruesa) & $680,6 \mathrm{a}$ & 725,0 a & $477,5 \mathrm{a}$ & $680,6 \mathrm{a}$ & $723,3 \mathrm{a}$ & $477,5 \mathrm{a}$ \\
\hline Crisantemo & $126,5 \mathrm{a}$ & $66,1 \mathrm{a}$ & $22,5 \mathrm{a}$ & $125,1 \mathrm{a}$ & $66,1 \mathrm{a}$ & $22,5 \mathrm{a}$ \\
\hline $\begin{array}{l}\text { Crisantemo } \\
\text { (gruesa) }\end{array}$ & $808,0 \mathrm{~b}$ & 2379,6 a & $2401,1 \mathrm{a}$ & $807,0 \mathrm{~b}$ & $2377,3 \mathrm{a}$ & $2401,1 \mathrm{a}$ \\
\hline Gladiola & $1908,8 \mathrm{a}$ & 852,3 ba & $100,0 \mathrm{~b}$ & $1898,0 \mathrm{a}$ & 852,3 ba & $100,0 \mathrm{~b}$ \\
\hline Gladiola (gruesa) & $604,1 \mathrm{c}$ & $2500,0 \mathrm{~b}$ & $3669,0 \mathrm{a}$ & $604,1 \mathrm{c}$ & 2491,6 b & $3661,6 \mathrm{a}$ \\
\hline Nardo & $203,3 \mathrm{a}$ & $111,4 \mathrm{ba}$ & $11,5 \mathrm{~b}$ & $184,5 \mathrm{a}$ & $111,4 \mathrm{a}$ & $11,5 \mathrm{a}$ \\
\hline Nardo (gruesa) & $83,5 \mathrm{~b}$ & $169,8 \mathrm{ba}$ & 270,6 a & $83,0 \mathrm{a}$ & $169,8 \mathrm{a}$ & $269,6 \mathrm{a}$ \\
\hline Nube & 672,3 a & 623,3 a & $536,8 \mathrm{a}$ & 660,3 a & 613,3 a & $527,3 \mathrm{a}$ \\
\hline Rosa & $449,0 \mathrm{a}$ & $135,2 \mathrm{ba}$ & $24,0 \mathrm{~b}$ & $358,0 \mathrm{a}$ & $134,4 \mathrm{a}$ & $24,0 \mathrm{a}$ \\
\hline Rosa (gruesa) & $262,8 \mathrm{a}$ & $675,1 \mathrm{a}$ & $696,5 \mathrm{a}$ & $258,0 \mathrm{a}$ & $665,1 \mathrm{a}$ & 683,3 a \\
\hline Zempoalxochitl & $5059,3 \mathrm{a}$ & $1475,5 \mathrm{~b}$ & $1009,5 \mathrm{~b}$ & $5010,0 \mathrm{a}$ & $1474,1 \mathrm{~b}$ & $988,5 \mathrm{~b}$ \\
\hline
\end{tabular}

Fuente: elaboración propia, 2015

Tabla 2. Análisis comparativo entre sexenios para el volumen de la producción y el valor de la producción asociados a los grupos de cultivos ornamentales.

\begin{tabular}{|c|c|c|c|c|c|}
\hline \multirow[b]{2}{*}{ Sexenio } & \multicolumn{2}{|c|}{ Volumen de la Producción } & \multicolumn{3}{|c|}{ Valor de la Producción } \\
\hline & $1995-2000$ & & $1995-2000$ & 2001-2006 & 2007-2012 \\
\hline Grupo & Valor Promedio & & & Valor Promedio & \\
\hline Agapando (Gruesa) & $15615,5 \mathrm{a}$ & $26837,8 \mathrm{a}$ & $555620,5 \mathrm{a}$ & $1304383,9 \mathrm{a}$ & $428802,9 \mathrm{a}$ \\
\hline Alelí & $1827,0 \mathrm{~b}$ & 2554,8 a & $313728,5 \mathrm{a}$ & $651836,5 \mathrm{a}$ & $333140,4 \mathrm{a}$ \\
\hline $\begin{array}{l}\text { Ave del Paraíso } \\
\text { (Gruesa) }\end{array}$ & $39266,6 \mathrm{a}$ & $81478,3 \mathrm{a}$ & $880154,0 \mathrm{a}$ & $1273375,4 \mathrm{a}$ & $1290251,1 \mathrm{a}$ \\
\hline Clavel (Gruesa) & $5573439,8 \mathrm{a}$ & $3361670,0 \mathrm{a}$ & $23444602,6 \mathrm{a}$ & 30601224,3 a & $27165980,4 \mathrm{a}$ \\
\hline Crisantemo & $1105,6 \mathrm{a}$ & $143,5 \mathrm{a}$ & $489828,5 \mathrm{a}$ & 247394,1 ba & $14935,8 \mathrm{~b}$ \\
\hline $\begin{array}{l}\text { Crisantemo } \\
\text { (Gruesa) }\end{array}$ & $4333260,8 \mathrm{a}$ & $10480262,0 \mathrm{a}$ & $35803458,0 \mathrm{~b}$ & 96444564,2 a & $102429431,0 \mathrm{a}$ \\
\hline Gladiola & $25708,8 \mathrm{a}$ & $3583,0 \mathrm{a}$ & $12155798,1 \mathrm{a}$ & $8720597,3 \mathrm{a}$ & $2529188,2 \mathrm{a}$ \\
\hline Gladiola (Gruesa) & $663110,5 \mathrm{~b}$ & $3863302,0 \mathrm{a}$ & $8805463,9 \mathrm{c}$ & $36006782,4 \mathrm{~b}$ & $62954373,4 \mathrm{a}$ \\
\hline Nardo & 2529,5 a & $144,5 \mathrm{~b}$ & $1418035,5 \mathrm{a}$ & $1071603,5 \mathrm{a}$ & $119610,75 a$ \\
\hline Nardo (Gruesa) & $108846,5 \mathrm{a}$ & $325381,6 \mathrm{a}$ & $1194564,5 \mathrm{a}$ & $2024021,2 \mathrm{a}$ & $3119813,9 a$ \\
\hline Nube & $5407,1 \mathrm{a}$ & $4291,6 \mathrm{a}$ & $778772,9 \mathrm{a}$ & $1211340,6 \mathrm{a}$ & $658360,6 \mathrm{a}$ \\
\hline Rosa & $6387,8 \mathrm{a}$ & $153,5 \mathrm{~b}$ & $4903669,5 \mathrm{a}$ & 989947,5 b & $68789,6 \mathrm{~b}$ \\
\hline Rosa (Gruesa) & $1550707,5 \mathrm{a}$ & $1032160,1 \mathrm{a}$ & 49227940,2 a & $35515416,7 \mathrm{ba}$ & $13863114,0 \mathrm{~b}$ \\
\hline Zempoalxochitl & $52603,1 \mathrm{a}$ & $8716,8 \mathrm{~b}$ & $8498317,6 \mathrm{a}$ & $4138087,1 \mathrm{~b}$ & $1497583,7 \mathrm{~b}$ \\
\hline
\end{tabular}


Al evaluar el coeficiente de variación se observa claramente alta heterogeneidad en los diferentes sexenios en relación con los cultivos evaluados. Destacando que la superficie sembrada asociada con el cultivo de agapando (gruesa), crisantemo, nardo, rosa y zempoalxochitl presentan un patrón de heterogeneidad en el tiempo evaluado. En cuanto a los cultivos de alelí y nube se detectó similitud entre los sexenios en relación con el área sembrada, área cosechada y volumen de producción (tabla 3 ).

Tabla 3. Coeficiente de variación entre sexenios para las especies estudiadas

\begin{tabular}{ccccccccccccc}
\hline & \multicolumn{1}{c}{ Sexenio 1 } & \multicolumn{1}{c}{ Sexenio 2 } & \multicolumn{4}{c}{ Sexenio 3 } \\
& SSe & SC & VP & VLP & SSe & SC & VP & VLP & SSe & SC & VP & VLP \\
\hline $\mathbf{1}$ & 46 & 46 & 64,3 & 145,2 & 30,4 & 30,4 & 118,5 & 114,5 & 15,2 & 8 & 3,3 & 9,7 \\
$\mathbf{2}$ & 11,9 & 11,9 & 17,1 & 70,2 & 1,9 & 1,9 & 9,3 & 93,9 & 25,1 & 26,2 & 28,5 & 28,8 \\
$\mathbf{3}$ & 33,2 & 32,6 & 51,8 & 38,8 & 48,1 & 48,1 & 42,6 & 38,1 & 2,5 & 3,4 & 13,3 & 32,8 \\
$\mathbf{4}$ & 30,1 & 30,1 & 52,2 & 41,1 & 2,1 & 2,3 & 24,8 & 26 & 9,9 & 9,9 & 7,1 & 24,3 \\
$\mathbf{5}$ & 29,4 & 30,7 & 29,9 & 35,4 & 66,3 & 66,3 & 87,7 & 101,5 & 135,1 & 135 & 140 & 137,1 \\
$\mathbf{6}$ & 82,6 & 82,8 & 87,2 & 79,6 & 3,2 & 3,2 & 17,4 & 19,4 & 3,8 & 3,8 & 13,9 & 31,9 \\
$\mathbf{7}$ & 4,6 & 4,8 & 9,1 & 15,8 & 109,2 & 109,2 & 128,8 & 94,4 & - & - & - & - \\
$\mathbf{8}$ & 40,3 & 40,3 & 34,4 & 42,5 & 35,9 & 36,2 & 85,2 & 35,7 & 3,3 & 3,2 & 6,9 & 6,2 \\
$\mathbf{9}$ & 23,5 & 22,2 & 26,8 & 42,9 & 74,8 & 74,8 & 82,9 & 77,6 & 92,2 & 92,2 & 63,1 & 129,9 \\
$\mathbf{1 0}$ & 102,8 & 104 & 100,6 & 131,9 & 59,1 & 59,1 & 62 & 67,8 & 11,5 & 10,8 & 8,2 & 27,2 \\
$\mathbf{1 1}$ & 13 & 12,6 & 11,8 & 38,8 & 13,6 & 15,3 & 16 & 67 & 9,9 & 9,7 & 11,3 & 19 \\
$\mathbf{1 2}$ & 27,8 & 29,5 & 15,6 & 10,8 & 144,2 & 145,4 & 172,7 & 171,8 & 117,8 & 118 & 92,5 & 86,4 \\
$\mathbf{1 3}$ & 24,3 & 22,8 & 39 & 69,6 & 33,7 & 34,3 & 53,9 & 59,9 & 9,4 & 8,4 & 18,3 & 26,3 \\
$\mathbf{1 4}$ & 30,8 & 30,5 & 31,3 & 39,5 & 28,8 & 29,9 & 36,6 & 49,1 & 38 & 39,2 & 39,6 & 38,1 \\
\hline
\end{tabular}

Fuente: elaboración propia, 2015.

1 agapando (gruesa); 2 alelí; 3 ave del paraíso (gruesa); 4 clavel (gruesa); 5 crisantemo; 6 crisantemo (gruesa); $\mathbf{7}$ gladiola; 8 gladiola (gruesa); $\mathbf{9}$ nardo; $\mathbf{1 0}$ nardo (gruesa); $\mathbf{1 1}$ nube; $\mathbf{1 2}$ rosa; $\mathbf{1 3}$ rosa (gruesa); 14 zempoalxochitl; SSe Superficie Sembrada; SC Superficie Cosechada; VP Volumen de Producción; VLP Valor de Producción.

El análisis de componentes principales estableció para la superficie sembrada que el primer factor se explica por la dinámica relacionada con los cultivos de: agapando (gruesa), alelí, ave del paraíso (gruesa), crisantemo, crisantemo (gruesa), gladiola, gladiola (gruesa), nardo, nardo (gruesa), rosa, rosa (gruesa) y zempoalxochitl siendo este conjunto de cultivos los que explican la mayor variabilidad existente. Para la superficie cosechada tuvo una similitud exacta a la superficie cosechada (tabla 4).

Tabla 4. Análisis de componentes principales por tema.

\begin{tabular}{|cc|c|c|c|c|c|c|c}
\hline & \multicolumn{3}{c}{ SSe } & \multicolumn{2}{c}{ SC } & \multicolumn{2}{c}{ VP } & \multicolumn{2}{c}{ VLP } \\
\hline $\mathbf{1}$ & 0,8232 & 0,30418 & 0,80826 & 0,31124 & 0,11488 & 0,74578 & 0,51062 & 0,69729 \\
$\mathbf{2}$ & 0,84102 & 0,25473 & 0,81814 & 0,26183 & 0,77196 & 0,28978 & 0,489 & 0,84112 \\
$\mathbf{3}$ & 0,9312 & 0,0911 & 0,93496 & 0,06347 & 0,85033 & $-0,10625$ & $-0,49555$ & 0,35321 \\
$\mathbf{4}$ & 0,0479 & $-0,80225$ & $-0,0166$ & $-0,73761$ & $-0,09479$ & 0,83457 & 0,02002 & 0,70229 \\
\hline $\mathbf{5}$ & $--0,79058$ & 0,22418 & $-0,80868$ & 0,29597 & $-0,83344$ & 0,32743 & 0,89116 & 0,03135 \\
$\mathbf{6}$ & 0,73226 & 0,42542 & 0,72271 & 0,44488 & 0,56958 & 0,67564 & $-0,25721$ & 0,83884 \\
$\mathbf{7}$ & $-0,93308$ & 0,01575 & $-0,94387$ & 0,05844 & $-0,88825$ & 0,19733 & 0,93459 & 0,25186 \\
$\mathbf{8}$ & 0,98307 & $-0,02634$ & 0,97321 & $-0,04697$ & 0,94951 & $-0,05409$ & $-0,83435$ & 0,46709 \\
$\mathbf{9}$ & $-0,90914$ & $-0,02893$ & $-0,87987$ & $-0,10885$ & $-0,88922$ & 0,2741 & 0,88325 & 0,17855 \\
\hline$\#$ & 0,72815 & $-0,54858$ & 0,711 & $-0,58429$ & 0,76963 & $-0,43826$ & $-0,76161$ & 0,0982 \\
\hline$\#$ & $-0,3967$ & 0,47192 & $-0,34692$ & 0,43678 & 0,42054 & 0,49053 & 0,57164 & 0,77566 \\
\hline$\#$ & $-0,93217$ & 0,23757 & $-0,88698$ & 0,23068 & $-0,94913$ & 0,04025 & 0,75579 & $-0,43654$ \\
\hline$\#$ & 0,81009 & 0,19679 & 0,81895 & 0,15613 & 0,37401 & 0,43307 & 0,40804 & $-0,2621$ \\
\hline$\#$ & $-0,78262$ & $-0,02257$ & $-0,75998$ & $-0,03853$ & $-0,81674$ & $-0,27641$ & 0,69254 & $-0,51067$ \\
\hline
\end{tabular}

Fuente: elaboración propia, 2015.

$\mathbf{1}$ agapando (gruesa); $\mathbf{2}$ alelí; $\mathbf{3}$ ave del paraíso (gruesa); 4 clavel (gruesa); 5 crisantemo; 6 crisantemo (gruesa); 7 gladiola; 8 gladiola (gruesa); 9 nardo; 10 nardo (gruesa); 11 nube; 12 rosa; 13 rosa (gruesa); 14 zempoalxochitl; SSe Superficie Sembrada; SC Superficie Cosechada; VP Volumen de Producción; VLP Valor de Producción.

En cuanto al volumen de la producción, las flores de alelí, ave del paraíso (gruesa), crisantemo, gladiola, gladiola (gruesa), nardo, nardo (gruesa), rosa y zempoalxochitl son en conjunto las que explican el mayor porcentaje de variabilidad multidimensional. En lo referente al valor de la producción se detectó que ave del paraíso (gruesa), crisantemo, gladiola, gladiola (gruesa), nardo, nardo (gruesa), rosa, rosa (gruesa) y zempoalxochitl son los cultivos que predominan en el análisis de componentes.

En cuanto a la aportación del valor de la producción de la floricultura al PIB agrario 
nacional se evidencia una estrecha relación con un comportamiento cíclico, fuertemente autocorrelacionado con una tendencia ascendente, sin tener influencia de los sexenios (figura 1).

Al analizar la dinámica del gasto anual en productos ornamentales por habitante, se encontró una tendencia hacia la baja en el período analizado, a diferencia del gasto en productos agropecuarios (figura 2).

Figura 1. Aportación del valor de la producción de la floricultura al producto interno bruto agrario nacional.

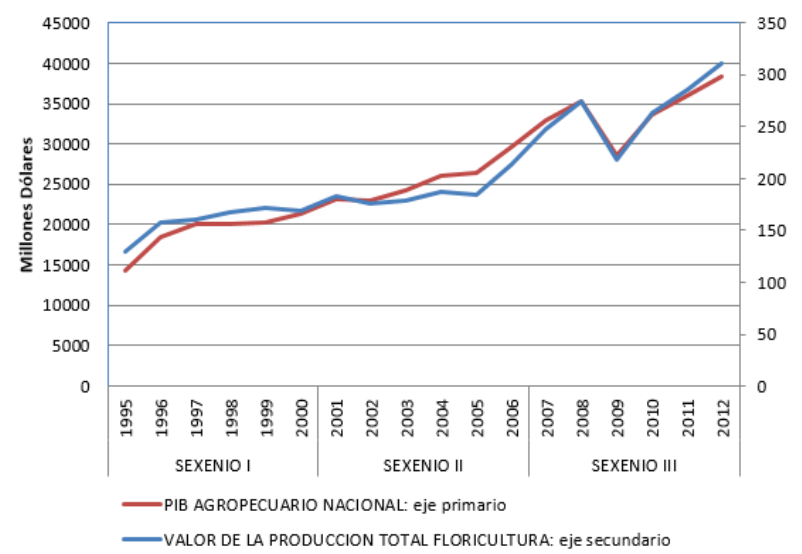

Fuente: Elaboración propia, 2015.

Figura 2. Comparación del gasto anual en productos agropecuarios y el gasto anual en ornamentales

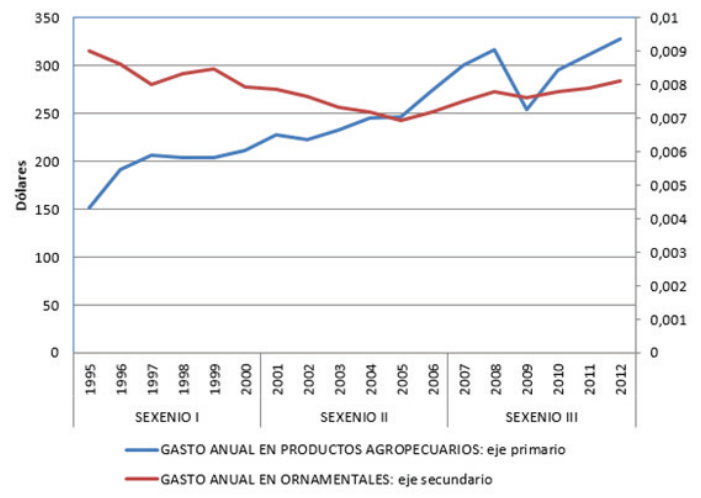

\section{Discusión de resultados}

Los resultados de este estudio indican que la superficie sembrada de Alelí y Gladiola (gruesa) tuvieron un incremento en el período analizado. Al respecto, otros estudios reportan que esta tendencia se debe a un incremento en las exportaciones a Estados Unidos ya que estas flores y en especial el alelí conforme se ha ido introduciendo con una mayor calidad han aumentado la demanda en este mercado y la gladiola incluso en los meses de invierno en los cuales su producción se dificulta (Monge, Martines, \& Bou, 1999). En cuanto a los cultivos de Gladiola, Nardo y Rosa, se encontró una disminución en la superficie sembrada. Al respecto, otros estudios han reportado que debido a la implementación de nuevas tecnologías en estos cultivos, ya no se están sembrando a cielo abierto sino en invernaderos (INIFAP, 2005).

Por otra parte, se encontró que la superficie cosechada para el caso de Alelí y Gladiola (gruesa) tuvo un incremento, especialmente en el último sexenio. El incremento creciente de la superficie cosechada en los sexenios analizados, indica un aumento en la importancia de la floricultura en los últimos años; paradójicamente, no se ha tenido en el período analizado, una política nacional de fomento al cultivo o exportación de ornamentales, situación que conlleva a pensar que el crecimiento se ha dado por un incremento en la demanda interna de este tipo de productos en el país. En este sentido, como el caso que explica Massieu, en México el mercado interno de flores y ornamentales es más importante que la exportación, pese a que México es uno de los países del mundo con más tratados de libre comercio firmados y que la política económica respecto al sector agropecuario destina los escasos incentivos de inversión pública a productos susceptibles de ser exportados (Massieu, 2010). 
En lo referente al volumen de la producción únicamente el cultivo de Nardo tuvo aumento en el período analizado. Esta situación puede ser debido a que el Nardo ha aumentado su demanda y hoy se cultiva en muchos Estados de la república por su agradable aroma y por el ciclo corto de cultivo (Torres, 2010).

En lo que respecta al valor de la producción el cultivo de Gladiola (gruesa) tuvo un aumento a lo largo del tiempo; debido al aumento en las exportaciones, especialmente de esta variedad, siendo los principales destinos Estados Unidos y Canadá (SAGARPA, 2012). El Crisantemo y Rosa (gruesa) por su parte presentaron una disminución en el valor de la producción a través de los diferentes sexenios; de acuerdo con otras investigaciones, esto se debe más al inicio del cultivo bajo cubierta o invernadero que lleva al descenso en el cultivo de las mismas flores a cielo abierto.

En cuanto a la aportación del valor de la producción de la floricultura al PIB agrario nacional, se encontró que no hubo influencia de los sexenios; este tipo de análisis comparativo permite la determinación de patrones de comportamiento sistemático para señalar presencia de ritmos de crecimiento (Escalante, Galindo \& Catalán, 2005). El comportamiento atípico del PIB en el tercer sexenio, se debió a la desaceleración de crecimiento de la economía mexicana (CDHCU, 2009), como consecuencia de la crisis económica acontecida en 2008-2009; causada debido a que la tasa de ahorro en los Estados Unidos cayó a cero y los norteamericanos comenzaron a tomar préstamos en gran escala de países como china, para sostener su consumo excesivo (Tanzi, 2010).

La dinámica del gasto anual en productos ornamentales por habitante, indica una tendencia hacia la baja en el período analizado, situación que puede estar relacionada con el incremento en el valor de las flores (FSOO, 2009); por cuanto la cultura mexicana siempre ha tenido una tendencia al consumo de flores durante todo el año. Por otro lado, otros estudios indican que en la comercialización de las flores inciden otros factores como la competencia con productos sustitutos y cambios en las preferencias por temporadas (Orozco, 2007), que también pueden afectar el gasto en este tipo de productos. Por el contrario, el gasto en productos agropecuarios forma un eslabón esencial para la alimentación de todas las personas (FAO, 2002).

\section{Conclusiones}

El área cultivada en flores en México se destaca por un mayor crecimiento en el último sexenio debido al crecimiento de las exportaciones, en especial del Alelí y Gladiola (gruesa). El cultivo de Nardo tuvo un incremento en la producción en el último sexenio, debido al incremento en su demanda por sus ventajas como el aroma y el ciclo corto de cultivo.

La aportación del valor de la producción de la floricultura al PIB agrario nacional tiene una tendencia ascendente, sin tener influencia de los sexenios, por lo cual es preciso contar con una visión que permita dimensionar la productividad y los beneficios de la floricultura en México, teniendo en cuenta la importancia de la política nacional para el desarrollo esta actividad. Por su parte el gasto anual en productos ornamentales por habitante, presenta una tendencia hacia la baja en el período analizado, lo cual indica la necesidad de implementar políticas para incrementar el consumo. 


\section{Referencias bibliográficas}

Castell Torres J.(2010). El nardo. Tomado de: http://www.mapama.gob.es/ministerio/ $\mathrm{pags/biblioteca/revistas/pdf \text {Hort/ }}$ Hort $1990 \quad 58 \quad 7$ 24.pdf

Centro de Estudios de las Finanzas Públicas de la H. Cámara de los Diputados. (2009). La Crisis Financiera de los Estados Unidos y su impacto en México. México: CEFP.

Escalante R., L. Galindo y H. Catalán. (2005). La evolución del producto del sector agropecuario mexicano, 1960-2002: Algunas regularidades empíricas. Revista Cuadernos de Desarrollo Rural 54 pp. 87-112.

Grammont, H. (2010) La evolución de la producción agropecuaria en el campo mexicano: Concentración productiva, pobreza y pluriactividad. Andamios 7 (13) pp.85-117.

Instituto Nacional de Investigaciones Forestales, Agrícolas y Pecuarias. (2005). Ciencia para el Desarrollo en Morelos. Publicación especial 41, pp.3-12.

Massieu, Y. (2010). El trabajo y los lujos de la tierra. Biotecnología y jornaleros en la agricultura globalizada de México. México: Juan Pablos Editor.

Monge A. Martínez A. y Bou M. (1999). Cultivo del aleli en invernadero para flor cortada. Valencia: Generalitat valenciana.

Morales S. (2011). Construcción de indicadores agregados para la toma decisiones financieras sector de floricultura, en el Estado de México. (Tesis de maestría). Universidad Autónoma de México, México, D.F.

Organización de las Naciones Unidas para la AlimentaciónylaAgricultura.(2002).Agricultura mundial: hacia los años 2015/2030. Departamento de Desarrollo Económico y Social. Tomado de: http://www.fao.org/ docrep/004/y3557s/y3557s00.htm
Orozco Hernández M. E. (2007). Entre la competitividad local y la competitividad global: floricultura comercial en el Estado de México. Revista de Ciencias Sociales, 14 pp. $111-160$

Santana, I. (2011). Aspectos técnicos de producción y comercialización de la flor de complemento limonium (sinatum y latifolium spp) en el Estado de México. (Tesis de pregrado). Universidad Autónoma Agraria Antonio Narro. Saltillo: México.

Secretaria de Agricultura, Ganadería, Desarrollo Rural,PescayAlimentación.(2012).Garantizada la disponibilidad de flores para cubrir la demanda nacional, Delegación en el Distrito Federal, Boletín 012, pp.1-4.

Secretaria de Agricultura, Ganadería, Desarrollo Rural,PescayAlimentación.(2015).Productores mexicanos preparados para abastecer demanda de flores, comunicado de prensa Sagarpa, Num.105/15, p.1-2.

Tanzi, V. (2010, julio). La crisis financiera y económica de 2008-2009: Efectos fiscales $y$ monetarios.En XXXVII Seminario Internacional de Presupuesto Público, Madrid: España. Tomado de: http://www19. iadb.org/intal/intalcdi/PE/2011/08050.pdf 